\title{
Draft genome sequences of the type strains of Shigella flexneri held at Public Health England: comparison of classical phenotypic and novel molecular assays with whole genome sequence
}

Philip M Ashton', Kate S Baker ${ }^{2}$, Amy Gentle ${ }^{1}$, David J Wooldridge ${ }^{3}$, Nicholas R Thomson², Timothy J Dallman ${ }^{1}$ and Claire Jenkins ${ }^{* *}$

\begin{abstract}
Background: Public Health England (PHE) holds a collection of Shigella flexneri Type strains isolated between 1949 and 1972 representing 15 established serotypes and one provisional type, E1037. In this study, the genomes of all 16 PHE Type strains were sequenced using the Illumina HiSeq platform. The relationship between core genome phylogeny and serotype was examined.

Results: The most common target gene for the detection of Shigella species in clinical PCR assays, ipaH, was detected in all genomes. The type-specific target genes were correctly identified in each genome sequence. In contrast to the S. flexneri in serotype 5 strain described by Sun et al. (2012), the two PHE serotype 5 Type strains possessed an additional oac gene and were differentiated by the presence (serotype $5 b$ ) or absence (serotype 5a) of gtrX. The somatic antigen structure and phylogenetic relationship were broadly congruent for strains expressing serotype specific antigens III, IV and V, but not for those expressing I and II. The whole genome phylogenies of the 15 isolates sequenced showed that the serotype 6 Type Strain was phylogenetically distinct from the other $S$. flexneri serotypes sequenced. The provisional serotype E1037 fell within the serotype 4 clade, being most closely related to the Serotype 4a Type Strain.
\end{abstract}

Conclusions: The S. flexneri genome sequences were used to evaluate phylogenetic relationships between Type strains and validate genotypic and phenotypic assays. The analysis confirmed that the PHE S. flexneri Type strains are phenotypically and genotypically distinct. Novel variants will continue to be added to this archive.

Keywords: Shigella flexneri type strains, Next generation sequencing technology, Molecular serotyping

\section{Background}

Shigella flexneri is the predominant cause of shigellosis in the developing world [1], making appropriate subtyping tools for tracking S. flexneri epidemiology vital to global public health. The S. flexneri serotyping scheme differentiates isolates serologically based on the expression of the major type specific somatic antigen (I-VI) and common group factor antigens (3,4 designated $\mathrm{Y}$ and 7,8 designated $\mathrm{X}$ ) [2]. The common group factor

\footnotetext{
*Correspondence: claire.jenkins@phe.gov.uk

${ }^{1}$ Gastrointestinal Bacteria Reference Unit, Public Health England, 61 Colindale Ave, NW9 5HT London, England

Full list of author information is available at the end of the article
}

antigens account for the complex intra-serotype relationships. Currently, there are 15 established serotypes. Traditional S. flexneri serotyping is performed by slide agglutination using antiserum raised in rabbits against type specific and group factor antigens. Recently, Sun et al. [3] published a multiplex PCR approach for molecular serotyping of $S$. flexneri. This method differentiates the 15 accepted serotypes based on known differences in (i) their gtr genes encoding the type specific antigens I, II, IV, and V, group factor antigen 7,8 (X) and 1c (gtrI, gtrII, $g \operatorname{tr} I V, g \operatorname{tr} V, g \operatorname{tr} X$, and $g \operatorname{trIC}$ ) (ii) the oac gene that mediates O-acetylation modification in serotypes $1 \mathrm{~b}, 3 \mathrm{a}, 3 \mathrm{~b}$, and $4 \mathrm{~b}$ and (iii) the $w z x_{6}$ for detection of serotype 6. 
Public Health England (PHE) holds an historic collection of 16 S. flexneri Type strains isolated between 1949 and 1972. Strains belonging to this set have been used to produce standardised antiserum for the phenotypic serotyping scheme at PHE for over 60 years. To increase the utility of this collection, we report the draft whole genome sequences of the 16 PHE S. flexneri Type strains in order to facilitate a greater understanding of how whole genome phylogenies compare to typing data generated from diagnostic and molecular serotyping targets.

\section{Methods}

\section{Bacterial strains}

The 16 strains of S. flexneri analysed in this study are shown in Table 1. Strains used in this study were serotyped by slide agglutination using both commercially available monovalent antisera (Denka Seiken, Japan) and monoclonal antibody reagents (Reagensia AB, Sweden) and in-house antisera raised in rabbits [4] to all type specific somatic antigens and the group factor antigens. All strains were tested using the PCR serotyping assay described by Sun et al. [3].

\section{Genome sequencing and analysis}

Genomic DNA was isolated from an overnight culture using the Wizard kit (Promega, Madison, Wisconsin, USA) and was sequenced at the Wellcome Trust Sanger Institute (WTSI) and PHE. Paired end libraries where each pair was $100 \mathrm{bp}$ in length were generated on the Illumina Hiseq 2500 instrument (San Diego, California, USA). Resulting FASTQ reads were processed using Trimmomatic v0.27 [5] to remove bases with a PHRED score of less than 30 and read length less than $50 \mathrm{bp}$ after quality trimming. High quality reads were then mapped to the reference strain, S. flexneri serotype 2a strain 2457 T (AE014073.1) [6], using BWA v0.6.2 and Single Nucleotide Polymorphisms called using GATK v2.5.2 in Unified Genotyper mode [7]. Positions in the reference genome where GATK mapping quality was below 30 and genotyping quality was below 50 in any strain were excluded from further analysis. Single Nucleotide Polymorphisms (SNPs) were defined as the sub-set of high quality positions (MQ $>30, G Q>50)$ where the base identified varied from the reference position. De novo assembly was performed using Velvet v1.2.3 [8] with K-mer selected using VelvetK (Table 2) (http://www.vicbioinformatics.com/software.velvetk.shtml).

Table 1 Comparison of the phenotypic and genotypic serotyping

\begin{tabular}{|c|c|c|c|c|c|c|}
\hline \multicolumn{2}{|c|}{ Phenotypic results } & \multicolumn{2}{|c|}{ PCR results } & \multicolumn{3}{|c|}{ Genome sequencing results } \\
\hline $\begin{array}{l}\text { Agglutination } \\
\text { reactions }\end{array}$ & $\begin{array}{l}\text { Phenotypic } \\
\text { serotype [2] }\end{array}$ & $\begin{array}{l}\text { Genes detected using } \\
\text { PCR scheme [3]. }\end{array}$ & $\overline{\text { PCR serotype [3] }}$ & $\begin{array}{l}\text { Serotype derived from } \\
\text { genome sequence } \\
\text { according to PCR } \\
\text { scheme [3] }\end{array}$ & $\begin{array}{l}\text { Number of SNPs different } \\
\text { in the core regions } \\
\text { compared with } \\
\text { reference strain }\end{array}$ & $\begin{array}{l}\text { Proportion of } \\
\text { reference } \\
\text { mapped to }\end{array}$ \\
\hline Type I & $1 a$ & gtrl & $1 \mathrm{a}$ & $1 \mathrm{a}$ & 2549 & 0.88 \\
\hline $\begin{array}{c}\text { Type I + Group } \\
6\end{array}$ & $1 b$ & $g t r l+o a c$ & $1 b$ & $1 b$ & 2552 & 0.87 \\
\hline MASF 1c & 1c & $g t r l+g t r C$ & 1c & 1c & 2661 & 0.88 \\
\hline Type $\|+Y$ & $2 a$ & gtrll & $2 a$ & $2 a$ & 731 & 0.91 \\
\hline Type $\|+X$ & $2 b$ & $g t r l l+g \operatorname{tr} X$ & $2 b$ & $2 b$ & 2650 & 0.89 \\
\hline Type III + X & 3a & $g t r l l l+g \operatorname{tr} X$ & 3a & 3a & 6947 & 0.86 \\
\hline Type III +Y & $3 b$ & gtrlll & $3 b$ & $3 b$ & 6976 & 0.85 \\
\hline Type III + Y & $3 c$ & gtrlll & $\begin{array}{l}\text { 3c Not included in } \\
\text { the PCR scheme }\end{array}$ & $\begin{array}{l}\text { Not included in the } \\
\text { PCR scheme }\end{array}$ & 7030 & 0.85 \\
\hline Type IV + Y & $4 a$ & gtrlV & $4 a$ & $4 a$ & 2595 & 0.87 \\
\hline $\begin{array}{l}\text { Type IV + } \\
\text { Group } 6\end{array}$ & $4 b$ & gtrlV +oac & $4 b$ & $4 b$ & 2528 & 0.87 \\
\hline Type V+Y & $5 a$ & gtrV $+o a c$ & $\begin{array}{l}\text { 5a Not included in } \\
\text { the PCR scheme }\end{array}$ & $\begin{array}{l}\text { Not included in the } \\
\text { PCR scheme }\end{array}$ & 4388 & 0.89 \\
\hline Type V +X & $5 b$ & $g \operatorname{tr} V+o a c+g t r X$ & $\begin{array}{l}\text { 5b Not included in } \\
\text { the PCR scheme }\end{array}$ & $\begin{array}{l}\text { Not included in the } \\
\text { PCR scheme }\end{array}$ & 4294 & 0.88 \\
\hline Type VI & 6 & $w z x 6$ & 6 & 6 & 47787 & 0.74 \\
\hline $\begin{array}{l}\text { MASF IV-1 } \\
\text { E1037 }\end{array}$ & E1037 & $g t r l V+\mid p t-O$ & MASF IV-1 E1037 & $\begin{array}{l}\text { 4a with Ipt-O (opt) } \\
\text { gene }\end{array}$ & 3333 & 0.88 \\
\hline$x$ & $x$ & $\operatorname{gtr} X$ & $x$ & $x$ & 6937 & 0.84 \\
\hline$Y$ & $Y$ & No amplification & $Y$ & $Y$ & 752 & 0.92 \\
\hline
\end{tabular}


Table 2 Genome statistics for the S. flexneri genomes sequenced in this study

\begin{tabular}{ccccccc}
\hline Serotype & $\begin{array}{c}\text { Number of high quality } \\
\text { mapped reads }\end{array}$ & $\begin{array}{c}\text { Average coverage of S. flexneri } \\
\text { 2457 T reference }\end{array}$ & $\begin{array}{c}\text { Kmer used in } \\
\text { Velvet assembly }\end{array}$ & $\begin{array}{c}\text { N50 } \\
\text { contigs }\end{array}$ & $\begin{array}{c}\text { Ne novo assembly } \\
\text { genome size }\end{array}$ \\
\hline $1 a$ & 5593126 & 121.5896957 & 79 & 31660 & 526 & 4369471 \\
$1 b$ & 5332110 & 115.9154348 & 79 & 31344 & 387 & 4176066 \\
$1 c$ & 5593956 & 121.6077391 & 81 & 30176 & 425 & 4255254 \\
$2 a$ & 5541229 & 120.4615 & 79 & 33398 & 417 & 4262526 \\
$2 b$ & 4869600 & 105.8608696 & 77 & 32299 & 421 & 4328906 \\
$3 a$ & 4590499 & 99.79345652 & 77 & 33146 & 510 & 4601423 \\
$3 b$ & 4803610 & 104.4263043 & 79 & 33159 & 450 & 4494495 \\
$3 c$ & 5604902 & 121.8456957 & 81 & 33478 & 451 & 4495552 \\
$4 a$ & 5328467 & 115.8362391 & 81 & 33309 & 413 & 4267213 \\
$4 b$ & 5106835 & 111.0181522 & 79 & 34439 & 391 & 4188134 \\
$5 a$ & 5461876 & 118.7364348 & 79 & 29622 & 443 & 4362146 \\
$5 b$ & 5250432 & 114.1398261 & 79 & 29416 & 459 & 4354058 \\
6 & 6350211 & 138.0480652 & 85 & 20677 & 614 & 4401927 \\
E1037 & 15797791 & 343.4302391 & 93 & 36513 & 378 & 4350496 \\
X & 17982247 & 390.918413 & 93 & 33586 & 414 & 4324701 \\
\hline Y & 20943063 & 455.2839783 & 95 & 29717 & 447 & 4491024 \\
\hline
\end{tabular}

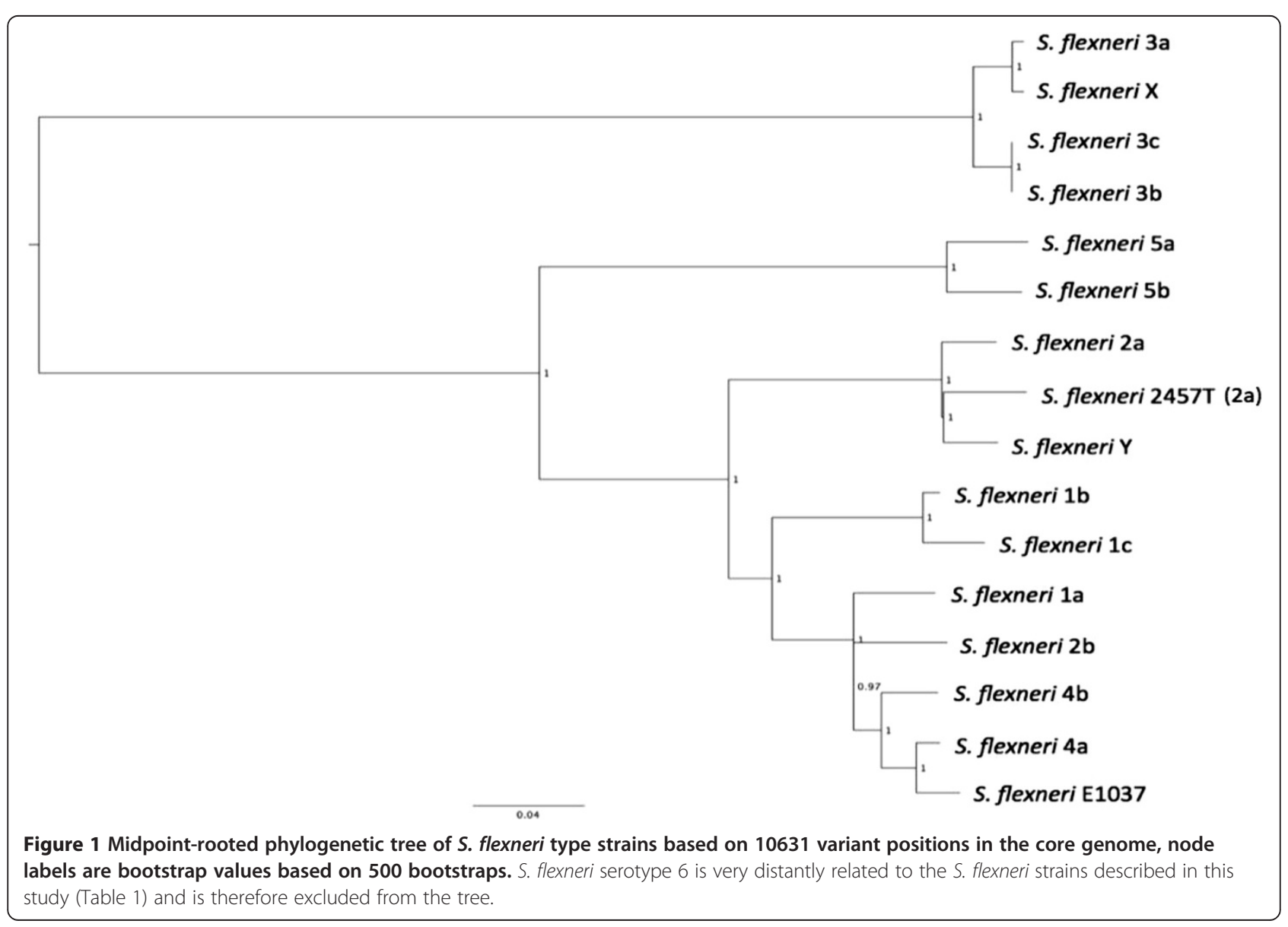


A maximum likelihood phylogenetic tree was drawn using MEGA v5.1 with 500 bootstraps based on an alignment of 10632 SNPs called against the $S$. flexneri serotype 2a strain $2457 \mathrm{~T}$ reference genome.

\section{Genomic data deposition}

Wellcome Trust Sanger Institute sequence data is available in the Short Read Archive under the following accession numbers (serotype): ERS088060 (1a); ERS088061 (1b); ERS088062 (1c); ERS088063 (2a); ERS088064 (2b); ERS088065 (3a); ERS088066 (3b); ERS088067 (3c); ERS0 88068 (4a); ERS088069 (4b); ERS088071 (5a); ERS088072 (5b); ERS088073 (6); ERS088074 (X); ERS088075 (Y); ERS088076 (E1037).

\section{Findings}

Mapping of the sequencing reads to the $4.6 \mathrm{Mbp}$ S. flexneri serotype 2a strain $2457 \mathrm{~T}$ reference genome resulted in 99-455 times coverage, with between 731 and 47787 SNPs compared to the reference genome (Table 1). De novo assembly resulted in an average N50 of 31621 with an average of 447 contigs (Table 2).

The phylogenetic relationships of the S. flexneri Type strains showed the somatic antigen structure and phylogenetic relationships were broadly congruent for strains expressing type specific antigens III, IV and V, but not I and II (Figure 1). In addition, serotype 3a was more closely related to the serotype $\mathrm{X}$ isolate than isolates expressing serotypes $3 \mathrm{~b}$ and $3 \mathrm{c}$. Serotype $3 \mathrm{c}$ was phylogenetically closely related to serotype $3 \mathrm{~b}$ but differed phenotypically as it failed to agglutinate with the 3,4 (y) group factor antigen. Serotype $3 \mathrm{c}$ is not longer included in the current serotyping scheme [3] as it is very rarely identified (nine isolates submitted to GBRU since 2004).

It has long been reported that the somatic $\mathrm{O}$ antigen of $S$. flexneri serotype 6 differs considerably from that of the other $S$. flexneri serotypes and that strains of $S$. flexneri serotype 6 resemble strains of $S$. boydii immunochemically [9]. Consistent with previous studies and phenotypic information, serotype 6 formed an out group from the other $S$. flexneri serotypes sequences (data not shown) [10] being more closely related to Shigella boydii CDC 3083-94 (GenBank: CP001063.1); differing by 47787 SNPs from $S$. flexneri 2a (Table 1) and approximately 7300 SNPs from $S$. boydii CDC 3083-94 (data not shown).

In 1972, colleagues in our laboratory reported a provisional new serotype, designated E1037, frequently submitted to PHE between 2004 and 2013 (276 isolates submitted to GBRU since 2004). Phylogenetically, E1037 is closely related to Serotype 4a (Figure 1). Other groups have supported the extension of the accepted classification scheme to include this novel type $[11,12]$.

The presence of key diagnostic and molecular serotyping genes was also determined. We confirmed the presence of the ipaH gene (the target gene for the detection of Shigella species in diagnostic PCR assays) in all the PHE Type strains. It was not possible to de novo assemble the complete ipaH gene in any strain analysed here due to the presence of multiple homologues of $i p a H$ in the genome. However, all 16 genomes showed the presence of the entire length of ipaH by either BLAST comparison of multiple contigs or mapping to the $S$. flexneri 2a 2457 T reference genome.

The molecular serotyping detailed in Sun et al. [3] correlated with the phenotypic data for all isolates tested (Table 1). The provisional type, E1037, was the only Type Strain to contain a copy of the plasmid-mediated seroconverting Ipt-O (opt) gene [12]. In contrast to the serotype 5 strain described by Sun et al. (2012) [3], both PHE serotype 5 Type strains encoded an additional oac gene which was intact according to de novo assembly and the presence of the oac gene was confirmed by PCR [3]. The $5 \mathrm{a}$ and $5 \mathrm{~b}$ serotypes were differentiable by the presence (serotype $5 \mathrm{~b}$ ) or absence (serotype $5 \mathrm{a}$ ) of $g \operatorname{tr} X$ (Table 1).

\section{Future directions}

The PHE S. flexneri Type strain data set has been used in the validation and evaluation of genotypic and phenotypic assays and has facilitated the study of phylogenetic relationships within this species during outbreak investigations (unpublished observations). Analysis of the genome sequences, in conjunction with the phenotypic serotyping data, provided new insights into this historic strain set. Comparisons with the PCR serotyping scheme highlighted the need to add novel variants [13] in order to maintain a comprehensive collection of relevant Type strains.

\section{Abbreviations \\ SNPs: Single nucleotide polymorphisms.}

\section{Competing interests}

The authors declare that they have no competing interests.

\section{Authors' contributions}

AG and CJ performed serology and DNA extraction. DW carried out the DNA sequencing. PA, KB, NRT and TJD analyzed the sequencing data. PA, KB, TJD and $\mathrm{CJ}$ contributed to the writing of the manuscript. All authors read and approved the final manuscript.

\section{Acknowledgements}

The authors would like to acknowledge the support of Kathie Grant, Catherine Arnold, Jonathan Green and PHE NGS Implementation Group.

\section{Author details}

Gastrointestinal Bacteria Reference Unit, Public Health England, 61 Colindale Ave, NW9 5HT London, England. 'Wellcome Trust Sanger Institute, Genome Campus, Hinxton CB10 1SA, UK. ${ }^{3}$ Department for Bio-analysis and Horizon Technologies, Next Generation Sequencing, Public Health England, 61 Colindale Ave, London NW9 5HT, England.

Received: 17 February 2014 Accepted: 23 March 2014

Published: 31 March 2014 


\section{References}

1. Kotloff KL, Nataro JP, Blackwelder WC, Nasrin D, Farag TH, Panchalingam S, Wu Y, Sow SO, Sur D, Breiman RF, Faruque AS, Zaidi AK, Saha D, Alonso PL, Tamboura B, Sanogo D, Onwuchekwa U, Manna B, Ramamurthy T, Kanungo S, Ochieng JB, Omore R, Oundo JO, Hossain A, Das SK, Ahmed S, Qureshi S, Quadri F, Adegbola RA, Antonio M, et al: Burden and aetiology of diarrhoeal disease in infants and young children in developing countries (the Global Enteric Multicenter Study, GEMS): a prospective, case-control study. Lancet 2013, 382(9888):209-222.

2. Ewing WH, Carpenter KP: Recommended designations for the subserotypes of Shigella flexneri. Int I Syst Bacteriol 1966, 16:145-149.

3. Sun $Q$, Lan R, Wang Y, Zhao A, Zhang S, Wang J, Wang Y, Xia S, Jin D, Cui Z, Zhao H, Li Z, Ye C, Zhang S, Jing H, Xu J: Development of a multiplex PCR assay targeting $\mathrm{O}$-antigen modification genes for molecular serotyping of Shigella flexneri. J Clin Microbiol 2011, 49:3766-3770.

4. Gross RJ, Rowe B: Serotyping of Escherichia coli. In The Virulence of Escherichia coll: Reviews and Methods 1985; (Special Publication of the Society for General Microbiology no. 13): 345-360. Edited by Sussman M. London: Academic Press.

5. Lohse M, Bolger AM, Nagel A, Fernie AR, Lunn JE, Stitt M, Usadel B: RobiNA: a user-friendly, integrated software solution for RNA-Seq-based transcriptomics. Nucleic Acids Res 2012, 40(Web Server issue):W622-7.

6. Wei J, Goldberg MB, Burland V, Venkatesan MM, Deng W, Fournier G, Mayhew GF, Plunkett G 3rd, Rose DJ, Darling A, Mau B, Perna NT, Payne SM, Runyen-Janecky L, Zhou S, Schwartz DC, Blattner FR: Complete genome sequence and comparative genomics of Shigella flexneri serotype 2a strain 2457 T. Infect Immun 2003, 71:2775-2786.

7. DePristo MA, Banks E, Poplin R, Garimella KV, Maguire JR, Hartl C, Philippakis AA, del Angel G, Rivas MA, Hanna M, McKenna A, Fennell TJ, Kernytsky AM, Sivachenko AY, Cibulskis K, Gabriel SB, Altshuler D, Daly MJ: A framework for variation discovery and genotyping using next-generation DNA sequencing data. Nat Genet 2011, 43:491-498.

8. Zerbino DR, Birney E: Velvet: algorithms for de novo short read assembly using de Bruijn graphs. Genome Res 2008, 18:821-829.

9. Slopek S, Mulczyk M: Concerning the classification of Shigella flexneri 6 bacilli. Arch Immunol Ther Exp (Warsz) 1967, 15:600-603.

10. Yang J, Nie H, Chen L, Zhang X, Yang F, Xu X, Zhu Y, Yu J, Jin Q: Revisiting the molecular evolutionary history of Shigella spp. J Mol Evol 2007, 64:71-79.

11. Pryamukhina NS, Khomenko NA: Suggestion to supplement Shigella flexneri classification scheme with the subserovar Shigella flexneri 4c: phenotypic characteristics of strains. J Clin Microbiol 1988, 26:1147-1149.

12. Sun Q, Knirel YA, Lan R, Wang J, Senchenkova SN, Jin D, Shashkov AS, Xia S, Perepelov AV, Chen Q, Wang Y, Wang H, Xu J: A novel plasmid-encoded serotype conversion mechanism through addition of phosphoethanolamine to the O-antigen of Shigella flexneri. PLoS One 2012, 7:e46095.

13. Sun Q, Lan R, Wang J, Xia S, Wang Y, Wang Y, Jin D, Yu B, Knirel YA, Xu J: Identification and characterization of a novel Shigella flexneri serotype $Y v$ in China. PLoS One 2013, 8:e70238.

doi:10.1186/1757-4749-6-7

Cite this article as: Ashton et al:: Draft genome sequences of the type strains of Shigella flexneri held at Public Health England: comparison of classical phenotypic and novel molecular assays with whole genome sequence. Gut Pathogens 2014 6:7.

\section{Submit your next manuscript to BioMed Central and take full advantage of:}

- Convenient online submission

- Thorough peer review

- No space constraints or color figure charges

- Immediate publication on acceptance

- Inclusion in PubMed, CAS, Scopus and Google Scholar

- Research which is freely available for redistribution

Submit your manuscript at www.biomedcentral.com/submit
C Biomed Central 